\title{
Stiffman syndrome: a rare paraneoplastic disorder?
}

\author{
D E Bateman, R O Weller, P Kennedy
}

\begin{abstract}
An unusual case of the stiffman syndrome, associated with an oat cell carcinoma of the bronchus, is reported. Pathological examination showed that it was due to an encephalomyelitis similar to that seen in paraneoplastic disorders. This suggests that atypical cases of the stiffman syndrome may occasionally be paraneoplastic.
\end{abstract}

The stiffman syndrome is a disorder of continuous muscle rigidity and painful muscle spasms, originally described by Moersch and Woltman. ${ }^{1}$ Most commonly it is a chronic benign disorder ${ }^{2}$ possibly of autoimmune basis due to production of an antibody to GAD. ${ }^{3}$ Pathology is available from only a single case of this type ${ }^{4}$ and no central nervous system abnormality was found. Four cases of a different type of stiffman syndrome have been described..$^{5-8}$ Although the initial clinical picture is similar, the disorder is progressive leading to death within a year or two. Neuropathology of these cases showed an encephalomyelitis of unknown cause.

We report a further case which was associated with oat cell carcinoma of the bronchus. On the basis of this case and other reasons presented in the discussion we suggest that stiffman syndrome of this type may be a rare paraneoplastic disorder.

\section{Case history}

The patient was a 71 year old retired gardener. He gave a six month history of progressive aching and stiffness of both legs and the right arm. By the time he was admitted to hospital he required assistance to walk and was unable to straighten or elevate his right arm. Involuntary painful flexor spasms of the arm occurred, causing a "red hot knife" like sensation in it. Spontaneous and touch sensitive flexor spasms of the legs were troublesome at night. A few weeks before admission he noticed similar stiffness and ache in the left arm. There were no sensory symptoms or sphincter disturbance. The patient had been a heavy smoker until eight years previously.

General examination showed a thin wasted man whose right arm was permanently flexed at the elbow to $90^{\circ}$. Higher mental function was normal. The only abnormality of the cranial nerves was nystagmus on lateral gaze.
There was continuous spasm of the right biceps, pectoralis major and latissimus dorsi so that the arm could not be passively abducted at the shoulder or extended at the elbow. Touching the right arm caused further painful increase in the flexor spasm. There was plastic increase in tone in the left arm. The intrinsic muscles of the right hand were wasted with some occasional fasciculation. There was no weakness in the arms apart from grade 4 weakness of the intrinsic muscles of the right hand and the left deltoid. The upper limb reflexes were absent. There was severe plastic increase in tone in the lower limbs but no weakness. The knee jerks were brisk, the ankle jerks absent. The plantar response was unassessable due to flexor withdrawal. Sensory testing showed intact light touch, pinprick and joint position sense but vibration sense was absent to the iliac crests.

Chest radiograph showed an ill defined mass in the right mid zone. CT guided biopsy of this lesion showed that it was an oat cell carcinoma of the bronchus. CT brain scan showed mild cerebellar and cortical atrophy. Cervical myelogram showed no abnormality. The CSF was normal in all respects with no oligoclonal bands.

The patient was given chemotherapy for the oat cell carcinoma. Lioresal was partially effective in relieving his spasms. The patient's general condition deteriorated rapidly and he died of bronchopneumonia a few weeks after the start of his chemotherapy without any change in his neurological condition.

\section{Necropsy findings}

Necropsy was performed at Jersey General Hospital on 15 March 1988. Both lungs showed extensive consolidation but no residual tumour was found. Parabronchial lymph nodes were largely replaced by small cell carcinoma (oat cell carcinoma). The brain weighed 1370 grams and apart from a small lesion consisting of small blood vessels in the left occipital lobe, $1 \mathrm{~cm}$ posterior to the splenium, was macroscopically normal. There was no evidence of metastatic tumour. The spinal cord was macroscopically normal.

\section{Histology}

There was a mild degree of perivascular lymphocytic cuffing in the putamen and a small number of haemosiderin-laden macrophages were seen around vessels in the caudate nucleus, putamen and globus pallidus; the 
small lesion in the left occipital lobe was seen to be capillary telangiectasia on histology. A small focus of microglial proliferation was seen in the end plate region of the left hippocampus. No other histological abnormality was seen in the cerebal hemispheres, pons, midbrain, and cerebellum.

Sections of the lower medulla at the level of the cuneate and gracile nuclei showed a mild lymphocytic infiltration of the leptomeninges. Accumulation of lymphocytes in the perivascular spaces was seen around arteries but more prominently around the veins in both the grey and white matter areas. Many of the larger perforating vessels were surrounded by lymphocytes. Occasional microglial nodules were present in the white matter.

The spinal cord at C6, T1, midthoracic, and L1 levels showed widespread but thin perivascular accumulations of lymphocytes particularly around the radial perforating vessels in the white matter at all levels; lymphocytes were present around blood vessels in the anterior horns of the grey matter, sometimes more prominent on one side than the other but almost symmetrical at T1. No anterior horn cell loss was detected but there were a few axon balloons at most levels of the cord in the anterior horns of the grey matter. There was some reduction in the axons in the lateral corticospinal tracts and in the cuneate tracts of the dorsal columns at all levels of the cord examined. Mild lymphocyte cuffing was present around vessels in the leptomeninges at all levels. An increase in corpora amylacea was observed in the gracile tracts.

Some $80 \%$ of the perivascular lymphocytes expressed B-cell markers using the antibody MB2 (Eurodiagnostics); the other 20\% did not express either MB2 or the T cell marker MT1. Very few macrophages were identified around vessels using the MAC antibody.

Sections of the femoral nerve showed no abnormality and, in particular, no perivascular lymphocyte cuffing.

\section{Discussion}

The stiffman syndrome in this patient was temporally associated with the discovery of the oat cell carcinoma and exhaustive investigation failed to reveal any of the other known causes. The pathological findings were typical of encephalomyelitis associated with cancer. ${ }^{89}$ The clinical and pathological evidence strongly suggest therefore that the disorder was paraneoplastic.

The four cases described previously ${ }^{5-8}$ were very similar clinically to this case, presenting with painful stiffness and stimulus sensitive spasms. There are reasons for suggesting that these other cases could have been associated with undiscovered neoplasms. The pathology in all four was very similar to this case, being characteristic of encephalomyelitis associated with cancer. One of these cases also had perivascular infiltration in the hippocampus, ${ }^{5}$ an area typically affected in paraneoplastic limbic encephalitis. Though the clinical syndrome was not evident in life, often in cases of paraneoplastic encephalomyelitis the pathological changes may be more widespread than is evident clinically..$^{910}$ One case also had opsoclonus and myoclonus, a well recognised paraneoplastic syndrome. ${ }^{11}$ The pathological and clinical evidence suggests therefore that these cases could have been paraneoplastic although no neoplasm was found. Henson and Urich note that the lung cancer growth may be extremely small and escape detection at necropsy and explain why it may not have been discovered in these other cases. ${ }^{10}$

Clinically the myelitis associated with cancer is usually evident as an anterior horn cell disturbance or dorsal column loss due to a dorsal root ganglion lesion. ${ }^{10}$ This case together with the other four cases suggest that occasionally the myelitis may manifest as the stiffman syndrome. Possibly a cross reacting antibody to GAD may rarely occur. The development of a progressive stiffman syndrome in an older patient, especially if associated with a brainstem disorder should lead to investigation for an occult oat cell carcinoma, particularly if the patient has no evidence of any autoimmune disorder.

MAC antibody was provided by Dr D B Jones, Department of Pathology, Southampton University.

1 Moersch FP, Woltman HW. Progressive fluctuating muscular rigidity and spasm ("stiffman syndrome"): report of a case and some observations in 13 other cases. Mayo Clin Proc 1956;31:421-7.

2 Layzer RB. Stiffman Syndrome-an autoimmune disease? New Eng J Med 1988;318:1060-1.

3 Solimena M, Folli F, Denis-Donni S, et al. Autoantibodies to glutamic acid decaboxylase in a patient with stiffman syndrome, epilepsy, and type 1 diabetes mellitus. New Eng J Med 1988;318:1012-20.

4 Trethowan WH, Allsop JL, Turner B. The stiffman Syndrome. Arch Neurol 1960;3:448-56.

5 Kasperek S, Zebrowski S. Stiffman Syndrome and encephalomyelitis. Arch Neurol 1971;24:22-30.

6 Lhermitte F, Chain F, Escourelle R, et al. Un nouveau cas de contracture tetaniforme distinct du stiffman syndrome. Rev Neurol 1973;128:3-21.

7 Whitely AM, Swash M, Urich H. Progressive encephalomyelitis with rigidity. Brain 1976;99:27-42.

8 Howell DA, Lees AJ, Toghill PJ. Spinal internuncial neurones in encephalomyelitis with rigidity. $J$ Neurol Neurosurg Psychiatry 1979;42:773-85.

9 Henson RA, Lovell Hoffman H, Urich H. Encephalomyelitis with carcinoma. Brain 1965;88:449-64.

10 Henson R, Urich H. Cancer and the Nervous System. Oxford: Blackwell, 1982:332-6.

11 Sheinman BD, Gawler J. Opsoclonus and polmyoclonia complicating oat cell carcinoma of the bronchus. Postgrad Med J 1982;58:704-5. 